\title{
Estrogen Activation of CaM Kinases and Transcription Is Blocked by Vitamin D in MCF-7 Breast Cancer Cells
}

\author{
John M. Schmitt*, Jessica Magill, Amanda Ankeny, Renee Geck, Jessica Milligan, Hannah \\ McFarland, and Erica Rice \\ From the Biology Department, George Fox University, 414 N. Meridian St, Newberg, OR 97132 \\ Conflict-of-interest statement: All authors declare no conflict of interest related to this publication. \\ Contract grant sponsor: M.J. Murdock Charitable Trust \\ Contract grant numbers: 2009261 \& 2011267 \\ Email: jschmitt@georgefox.edu
}

\begin{abstract}
Calcium/calmodulin-dependent protein kinase (CaM Kinase) proteins are targets of hormones and growth factors and regulate cancer cell growth, apoptosis and migration. The hormone estrogen (E2) utilizes CaM Kinases to activate the Extracellular-signal regulated kinase (ERK) leading to MCF-7 breast cancer cell growth. The hormone Vitamin D (Vit D) may inhibit breast cancer cell growth however the cellular mechanisms of Vit D action remain to be elucidated. Within the present study we provide data that E2 stimulation of MCF-7 cells activates CaM Kinase Kinase (CaM KK), CaM KI, and ERK and the transcription factors Elk-1 and SRF. E2 treatment of MCF-7 cells potently stimulated Elk-1/SRF directed transcription of SRE-luciferase reporters. E2 activation of ERK, Elk-1, SRF and SRE-dependent luciferase activities were blocked by treating cells with the CaM KK inhibitor, STO-609, and the ERK inhibitor, U0126. Moreover, siRNAs directed against CaM KK and ERK blocked transcription factor phosphorylation and luciferase activity. Treatment of cells with Vit D, the hormone Epinephrine (Epi), or Forskolin, prevented E2 activation of CaM KK and ERK. Interestingly, Vit D promoted a PKA-dependent phosphorylation and inhibition of CaM KK as well as its association with 14-3-3. Epi and Vit D treatment of cells blocked the ability of E2 to active Elk-1 and SRE-luciferase activity. This data suggests an important role for CaM KK and ERK in regulating transcription downstream of E2 in MCF-7 cells. Our results also suggest that Vit D treatment of MCF-7 cells utilizes a unique PKA-dependent mechanism to block E2 activation of CaM KK, ERK and transcription.
\end{abstract}

Keywords and Abbreviations: Estrogen (E2); CaM KK, calcium/calmodulin-dependent protein kinase kinase; ERK, extracellular-regulated protein kinase; vitamin D (Vit D); PKA, protein kinase A ; Elk-1

\section{Introduction}

Breast cancer is a major cause of worldwide mortality and according to the Centers for Disease Control and Prevention more than 200,000 new cases are diagnosed each year in the United States alone. Epidemiologic data suggests that the incidence of breast cancer in the United States is higher in latitudes above $40^{\circ}$ such as the Pacific Northwest [1]. Numerous genetic and ecologic factors may contribute to these rates including sunlight exposure and diet. Garland et al. suggested that there was an inverse association between sunlight exposure and breast cancer mortality in the United States [2]. A possible explanation for this observation is that ultraviolet light from the sun stimulates the body to synthesize the active form of the hormone vitamin $\mathrm{D}$ (Vit D) also known as $1,25(\mathrm{OH})_{2} \mathrm{D}_{3}$ [3]. Consistent with this hypothesis, several clinical studies have shown that women who experience rare sun exposure or Vit D deficiency are at a significantly increased risk of developing breast cancer (reviewed in $[1,3,4]$ ). Mice that are rendered Vit D deficient or lacking Vit D receptor (VDR) demonstrate accelerated cancer development [5-7]. Moreover, a recent study has suggested that polymorphisms in human VDR may also facilitate breast cancer progression [8]. Upon Vit D binding to target VDRs it can regulate various cellular and physiologic functions using both rapid non-genomic and genomic mechanisms [9-12]. Vit D 
supplementation is currently being evaluated in clinical trials for breast cancer treatment however the precise cellular mechanisms of Vit D action remain to be elucidated.

Vit D has been shown to inhibit melanoma proliferation while have no effect on normal melanocytes $[13,14]$. Likewise, Vit D may regulate the growth of myocytes, chondrocytes, hepatocytes and osteoblasts as well as enhance apoptosis in certain cancer cells and may antagonize prostate and breast cancer cell proliferation [9, 12, 15-19]. Multiple research groups have demonstrated and characterized the presence of functional VDRs on breast cancer cells including the MCF-10A, MCF-7 and MDA-MB-231 types [9, 20-22]. One type of VDR, 1,25D 3 -MARRS, functions as a rapid non-genomic signaling receptor for Vit D and was recently shown to inhibit MCF-7 and MCF-10 growth however the signaling pathways downstream of VDR were not examined [9]. Vit D and VDRs have been proposed to regulate numerous intracellular molecules and signaling pathways including cAMP, PKA, PLC, calcium, Src, JNK, and the Extracellular-Regulated Protein Kinase (ERK) [12, 23-28]. ERK is found in nearly all cell types throughout the body and regulates numerous transcription and translation factors as well as promote cell growth and proliferation. Vit D was recently shown to block serum-stimulated activation of ERK in MCF-7 cells however the mechanism of the hormone to antagonize activation of ERK was not carefully evaluated [29].

Previous studies have shown that the ERK pathway is activated in the breast cancer cell line, MCF-7, by various stimuli including epidermal growth factor (EGF), prolactin, angiotensin, estrogen (E2) and carbachol [30-35]. Carbachol has been shown to initiate activation of the ERK signaling pathway through the $\mathrm{M}_{3}$-subtype of muscarinic G-protein Coupled Receptors (GPCRs) in MCF-7 cells [31]. Estrogen and carbachol treatment of MCF-7 cells cause a rapid increase in intracellular calcium concentration and calcium ions have a variety of intracellular binding targets including the protein calmodulin [31, 36-38]. The calcium/calmodulin complex binds and activates the Calcium/Calmodulindependent protein kinase (CaM Kinase) family of enzymes [39]. The CaM Kinase family of enzymes have an established role in regulating neuronal development and synaptic plasticity and includes the serine/threonine kinases CaM KK, CaM KII, CaM KI, and CaM KIV [38, 40]. Active CaM KK directly phosphorylates and activates CaM KI, CaM KIV, AKT and AMPK [40]. The significance of CaM Kinase signaling in cancer is rapidly emerging and has been suggested to participate in numerous cancers including lung, prostate, brain, and breast [39, 41]. The importance of CaM KK signaling is particularly noteworthy in cancer biology and investigating the various ways in which CaM KK functions in cancer cells needs further evaluation. We have shown that CaM KK working through CaM KI, ERK and the transcription factor, Elk-1, stimulates MCF-7 cell growth although we did not examine whether the hormones E2 or Vit D may regulate this pathway [31].

CaM KK and CaM KI regulation of ERK targets numerous cytoplasmic and nuclear proteins to control cellular function. For example, CaM KK working through ERK is able to trigger phosphorylation of the transcription factor CREB leading to the increased expression of Wnt-2 genes in neurons [42]. ERK can also phosphorylate p90Rsk, MSK1/2, MNK1/2, and Elk-1 among others [43-45]. Elk-1 is a transcription factor that dimerizes with serum response factor (SRF), binds DNA, and increases gene expression $[46,47]$. We have recently suggested that carbachol and E2 may increase Elk-1 phosphorylation and transcriptional activity via ERK in MCF-7 cells although the precise role for CaM KK on ERK and Elk-1 downstream of E2 was not investigated [31]. Moreover, the ability of Vit D to specifically regulate CaM KK, ERK and Elk-1 activity in MCF-7 cells has not been demonstrated. Vitamin D has been proposed to stimulate cAMP and PKA activity and, cAMP inhibits MCF-7 cell growth [11, 48, 49]. Therefore, several mechanisms exist that could explain Vit D antagonism of ERK activity including cAMP activation of PKA and the subsequent phosphorylation and inhibition CaM KK and the recruitment of 14-3-3 protein to CaM KK [50]. Another scenario could involve cAMP activation of the small $G$ protein Rap1 and blockade of Ras activation of Raf-1, the activation of inactivating phosphatases or PKA phosphorylation of Raf-1 [48]. Therefore, our goal was to carefully evaluate the ability of Vit D to inhibit CaM KK, ERK, and transcription downstream of E2 signaling in MCF-7 cells. In addition we sought to examine whether Vit D may utilize cAMP and PKA to mediate its inhibitory effects on CaM KK and ERK in our system. 


\section{$2 \quad$ Materials and Methods}

\subsection{Materials}

The following reagents were purchased from Sigma-Aldrich; -estradiol (Estrogen; E2), 1,25dihydroxyvitamin D3 (Vitamin D; Vit D), Epinephrine (Epi), Flag (M2) antibody and Flag-agarose conjugated beads, epidermal growth factor (EGF), and bovine pancreatic insulin. Antibodies that bind to phosphorylated ERK (pERK), total ERK (ERK 1/2), CaM KK, phosphorylated CaM KI (pCaM KI), Elk-1, SRF, 14-3-3, protein A/G plus, and HRP-conjugated secondary antibodies were all purchased from Santa Cruz Biotechnology. Scrambled control siRNAs and siRNAs directed against ERK were purchased from Santa Cruz Biotechnology. Antibodies that bind to phospho-PKA substrates (pCaM KK), phosphorylated CREB, phosphorylated Elk-1, phosphorylated SRF, and the cAMP ELISA assay kit were purchased from Cell Signaling Tech. Inc. STO-609 was purchased from Tocris. U0126 and Forskolin were purchased from EMD Millipore (Calbiochem). PVDF and SDS-PAGE reagents were purchased from Bio-Rad. Luminal reagent, for imaging, was purchased from Pierce Biotechnology. The transfection reagent, Lipofectamine 2000 was purchased from Life Technologies (Invitrogen). The Cignal SRE Reporter luciferase assay kit was purchased from SABiosciences (Qiagen Company). The luciferase assay substrate kit was purchased from Stratagene (Agilent Technologies).

\subsection{Cell Culture}

The MCF-7 cells were purchased from ATCC and cultured in Eagle's Minimum Essential Medium (EMEM), with $10 \%$ fetal bovine serum, L-glutamine at $37^{\circ}$, according ATCC guidelines. $70-80 \%$ confluent MCF-7 cells were placed in serum-free EMEM overnight, and then stimulated with the designated compounds for western blotting, immunoprecipitation, ELISA, or luciferase assays as

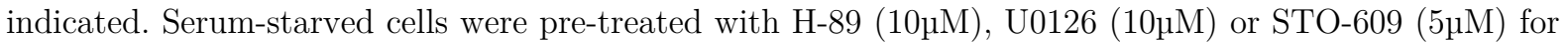
30 minutes prior to stimulation. Cells were pretreated for 5 minutes with Forskolin, Epinephrine, or Vitamin D prior to adding E2 for an additional 5 minutes, unless otherwise indicated. Cells were

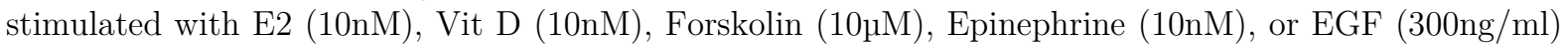
for 5 minutes per treatment unless otherwise indicated.

\subsection{Transfections}

Equivalent numbers of MCF-7 cells were plated and grown to 50-60\% confluency and then transfected with the indicated reagent using Lipofectamine 2000. The transfection reagents were prepared according to the manufacturer's guidelines using serum-free EMEM for 6 hours at $37^{\circ}$. Transfection media was then removed and replaced with complete media and the cells were allowed to recover 24 hours prior to serum-starvation and stimulation. siRNA transfections were carried out according to the manufacturer's guidelines and each plate received $20 \mathrm{mM}$ scrambled control siRNA or siRNA directed against ERK (Santa Cruz Biotechnology). Transfection of Flag-CaM KK isoforms (a generous gift from Monika Davare and Tom Soderling at OHSU), shRNA control plasmids, shRNA against CaM KK(a generous

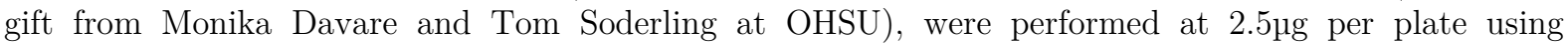
Lipofectamine. For luciferase assays, MCF-7 cells were transfected with SRE dual-luciferase formatted reporters (Qiagen) along with the indicated siRNA or shRNAs.

\subsection{Western Blotting and Immunoprecipitation}

MCF-7 cells were transfected or pretreated and then stimulated as indicated similar to previous studies [31]. The media was aspirated and the cells were lysed using ice-cold lysis buffer consisting of $10 \%$ glycerol, $1 \%$ NP-40, 50mM Tris- $\mathrm{HCl}$ at $\mathrm{pH} 7.4,200 \mathrm{mM} \mathrm{NaCl}, 2 \mathrm{mM} \mathrm{MgCl}$. along with the inhibitors PMSF $(1 \mathrm{mM})$, Aprotonin $(2 \mu \mathrm{g} / \mathrm{ml})$, Leupeptin $(1 \mu \mathrm{g} / \mathrm{ml})$ and Sodium Orthovanadate (1mM). Lysates were spun for 5 minutes at $4^{\circ}$ to pellet nucleotides and cellular debris. Equal volumes of supernatants were then aliquoted into a 96-well plate and quantified using the Bradford protein assay. Bovine serum albumin standards and samples were then read using a Bio-Rad 680 micro-plate reader. Equivalent protein amounts were then used for either immunoprecipitation or western blotting. $500 \mu \mathrm{g}$ of each 
lysate in normalized volumes were used for immunoprecipitation and incubated overnight at $4^{\circ}$ with their respective primary antibodies and agarose. Precipitates were washed $2 \mathrm{x}$ in ice-cold lysis buffer, run on SDS-PAGE gels and analyzed by western blotting. Lysates or precipitates were separated by SDSPAGE, blotted onto PVDF membranes using a Bio-Rad Turbo Transfer system and blocked in TBS Tween with 5\% milk for 1 hour at room temperature. Membranes were then probed overnight in TBS Tween with $5 \%$ Bovine serum albumin at $4^{\circ}$ using indicated primary antibodies, washed $4 \mathrm{x}$, and probed with the appropriate HRP-conjugated secondary antibody in TBS Tween with 5\% milk. Membranes were then incubated with luminol and imaged, scanned, densitized and quantified using a Kodak Image Station 2000R. Band intensities were normalized to loading control lysates and to the untreated control lanes and presented as fold phosphorylation, binding (association), or protein expression as appropriate.

\subsection{Luciferase Assay}

Luciferase activity was evaluated in MCF-7 cells using the cignal reporter assay from Qiagen. Cells were transfected with negative controls, positive controls, and SRE dual-luciferase formatted reporters (Qiagen) according to the manufacturer's guidelines using Lipofectamine 2000. To assay siRNA or shRNA knockdown cells were co-transfected with the indicated controls, siRNA, shRNA and the SRE dual-luciferase assay reporter as indicated. Following transfection, were allowed to recover in complete media for 24 hours and then serum-starved overnight and then pre-treated or stimulated with E2 $(10 \mathrm{nM})$, VitD (10nM), Epi (10nM) or the indicated combinations for 5 minutes. The cells were carefully washed 2x in PBS and lysed using luciferase assay lysis buffer from the luciferase assay kit according to the manufacturer's guidelines (Stratagene). Lysates were vortexed, centrifuged and equivalent amounts from each treatment sample were pipetted onto 96-well plates and immediately incubated with $100 \mu \mathrm{l}$ of luciferase substrate-assay buffer and analyzed using a GloMax-96 Microplate Luminometer (Promega). Luciferase activity was calculated and quantified based on control non-stimulated cells and presented as fold luciferase activity as indicated.

\subsection{ELISA Assay}

Cyclic AMP (cAMP) concentration was determined in MCF-7 cells using the cAMP XP assay kit according to the manufacturer's protocol (Cell Signaling Technology). Briefly, equivalent numbers of MCF-7 cells were plated, grown, serum-starved and stimulated as described in the cell culture section above. Cells were stimulated with the indicated compounds or hormones for 5 minutes and then rinsed with ice cold PBS and lysed using the cAMP XP lysis buffer on ice for 5 minutes. Cell lysates were briefly centrifuged to clear cell debris and a Bradford protein assay was performed to determine the protein concentration from each treatment. Equivalent amounts of cell lysates were then added to the cAMP assay 96-well plate and incubated at room temperature for 3 hours. Sample wells were washed 4x with wash buffer, incubated with TMB substrate for 30 minutes and read using a Bio-Rad 680 microplate reader at $450 \mathrm{~nm}$. Final cAMP concentrations were calculated, quantified and based on known control standard cAMP concentrations and presented in nano molar concentrations as indicated.

\section{$2.7 \quad$ Statistics}

To determine if statistically significant differences existed between specific experimental groups, Student $t$-tests were performed on the data. Significance levels were set at $0.05(95 \%)$ or higher and significant $P$ values are indicated in all figures as either "*" which indicates $P \leqslant 0.05$ or "**" which indicates $P \leqslant$ 0.01 .

\section{$3 \quad$ Results}

\subsection{Estrogen Treatment of MCF-7 Increases Transcription Factor Activation}

The serine/threonine kinases CaM KK and CaM KI have been shown to function upstream of ERK and several transcription factors in various cell types including breast cancer cells [31, 38, 40, 51-55]. 
Previous studies have demonstrated that estrogen (E2), carbachol, and epidermal growth factor (EGF) are capable of increasing ERK1/2 phosphorylation and activation in MCF-7 cells [31, 55]. In order to evaluate whether E2 can specifically activate CaM KK, MCF-7 cells were stimulated with a physiologic dose of E2 for 5 minutes and CaM KK activity was measured. E2 potently activated CaM KK and CaM KI, an effect that was not inhibited by the MEK inhibitory drug, U0126 (Fig. 1a). In contrast, E2 activation of CaM KK and CaM KI was completely blocked by pretreating cells with the selective CaM KK inhibitory compound STO-609 (Fig. 1a) [56, 57]. To determine if ERK1/2 are downstream of CaM KK and MEK in our system, MCF-7 cells were pretreated similarly to Fig. 1a and evaluated for ERK1/2 phosphorylation (pERK1/2). As can be seen in Fig. 1b pretreatment of cells with U0126 and STO-609 significantly blocked E2 activation of ERK. Elk-1 is a transcriptional target for ERK and is phosphorylated on serine 383 that triggers its association with SRF and DNA binding [46, 58, 59]. Elk-1 phosphorylation was elevated 3-fold with E2 stimulation in a manner that was significantly reduced by inhibiting ERK with U0126 and CaM KK with STO-609 (Fig. 1c). To ensure that STO-609 was acting to selectively inhibit CaM KK signaling in our system, MCF-7 cells were also stimulated with EGF in the presence or absence of STO-609. Consistent with other studies [31, 56, 57, 60], STO-609 did not block EGF-dependent stimulation of Elk-1 phosphorylation (Fig. 1d). Since CaM KK operates upstream of ERK and Elk-1, we also examined E2 activation of other ERK targets including CREB and SRF. Interestingly, E2 treatment of MCF-7 cells triggered a significant increase in both SRF and CREB phosphorylation at serine 103 and serine 133, respectively (Fig. 1e). E2 activation of SRF and CREB were both sensitive to pretreatment of MCF-7 cells with U0126 and STO-609 (Fig. 1e). Taken together the above data suggests that E2 is capable of activating ERK through CaM KK that leads to phosphorylation of several transcription factors including the early gene cell growth regulators Elk-1 and SRF.

Elk-1 and SRF are capable of forming heterodimers that may enhance their DNA binding at SRE elements and ability to regulate gene expression [46, 61]. Since Elk-1 and SRF are both phosphorylated in our system, we examined whether E2 stimulated the formation of a molecular complex between these proteins. E2 treatment of MCF-7 cells and immunoprecipitation of endogenous Elk-1 revealed a strong association with endogenous phosphorylated SRF protein (Fig. 2a). To directly evaluate E2's ability to regulate transcription through Elk-1 and SRF we transfected cells with a SRE-dual luciferase reporter plasmid and stimulated cells in the presence or absence of U0126 or STO-609. E2 increased SREdependent luciferase activity that was completely blocked by inhibiting ERK and CaM KK (Fig. 2b). To evaluate the significance of CaM KK and ERK signaling as well as the specificity of our observed effects, MCF-7 cells were transfected with siRNAs directed at CaM KK and ERK. E2 treatment of our cells stimulated both SRF and Elk-1 phosphorylation in a manner that was dependent upon CaM KK and ERK (Fig. 2c). To assess whether our siRNAs had significantly silenced their target protein expression in our system we examined expression of both CaM KK and ERK from our transfected cells. As can be seen in figures 2d and 2e we significantly reduced CaM KK and ERK protein expression, respectively, but not Actin in MCF-7 cells. We also examined the ability of CaM KK and ERK to mediate SRE-dependent gene expression by co-transfecting cells with the indicated siRNAs and the luciferase assay reporter. E2 treatment enhanced luciferase activity in our cells that was blocked by the presence of siRNA to either ERK or CaM KK (Fig. 2f). Based on these observations, E2 stimulates Elk1- and SRF-dependent gene expression through CaM KK and ERK in MCF-7 cells.

\subsection{Vitamin D and cAMP block CaM Kinase Signaling}

Previous studies have suggested that cAMP and PKA can inhibit CaM KK activity therefore we wanted to evaluate the ability of cAMP elevating agents to block CaM KK signaling in MCF-7 cells [50, 62, 63]. The compound Forskolin directly activates the enzyme adenylyl cyclase that converts ATP into cAMP and leads to activation of PKA. MCF-7 cells treated with Forskolin did not activate CaM KK, interestingly pretreatment of cells with Forskolin followed by E2 stimulation blocked CaM KK and CaM KI activation (Fig. 3a). Similarly, Forskolin did not activate ERK and significantly blocked E2's ability to stimulate ERK activation in our system (Fig. 3b). MCF-7 cells express Vitamin D receptors and the hormone, Vitamin D (Vit D), is capable of antagonizing ERK activation in certain cells although the mechanisms remain elusive. Based on this knowledge and the above data we examined if Vit D could block CaM KK signaling in our system. Similar to our previous results, E2 stimulated CaM KK and 
CaM KI activity an effect that was completely inhibited by Vit D (Fig. 3c). The downstream effects of Vit D on CaM KK were also determined and the results presented in figure 3d suggest that pretreatment of cells with Vit D block ERK activation downstream of E2. To further assess Vit D's blocking mechanism of CaM KK and ERK in our system we examined the duration and stability of phosphorylated ERK and Elk-1. E2 treatment of cells stimulated phosphorylation of both ERK and Elk1 up to 60 minutes an effect that was completely inhibited by Vit D (Fig. 3e). Vit D treatment alone did not appear to activate ERK or Elk-1 at the time points examined (Fig. 3e). To evaluate Vit D's effects on E2-induced transcriptional activity, SRE-dual luciferase reporter transfected cells were stimulated with E2, Vit D, or the combination. As can be seen in figure 3f, E2 stimulated an increase in Elk-1- and SRF-dependent luciferase activity an effect that was completely blocked by pretreatment with Vit D. Treatment of cells with Vit D alone had no effect on luciferase activity (Fig. 3f). MCF-7 cells express-adrenergic receptors that couple to cAMP/PKA and respond to the ligand epinephrine (epi) therefore we examined the ability of Epi to block E2 signaling in our system. Similar to Vit D pretreatment, Epi also blocked E2-stimulated luciferase activity and had no effect on its own (Fig. 3f). Taken together this data suggests that Vit D, Epi, and Forskolin inhibit CaM KK activation of ERK and transcription in our cells perhaps through cAMP and PKA.

CaM KK is a direct substrate for PKA and phosphorylated CaM KK binds to the cytosolic protein 14-3-3 and CaM KK activity is inhibited [50, 62, 63]. Based on the observation that Vit D inhibited CaM KK activity in our cells we wanted to examine whether Forskolin or Vit D could trigger PKA phosphorylation of CaM KK and increase its association with 14-3-3. E2 treatment of MCF-7 cells did not stimulate CaM KK phosphorylation by PKA however, Forskolin treatment in the presence and absence of E2 promoted a 4-5-fold increase in CaM KK phosphorylation (Fig. 4a). Vit D stimulation of MCF-7 cells also induced a significant increase in CaM KK phosphorylation by PKA as well as in the presence of E2 (Fig. 4b). To evaluate the association of CaM KK with 14-3-3 following PKA phosphorylation, we immunoprecipitated endogenous 14-3-3 from Forskolin- and Vit D-treated cells and examined the presence of endogenous CaM KK. Stimulation of cAMP and PKA with Forskolin triggered a strong increase in CaM KK binding to 14-3-3 and pretreating cells with Forskolin followed by E2 also revealed a significant association (Fig. 4c). E2 did not significantly affect 14-3-3 binding to CaM KK (Fig. 4c). Vit D stimulation of cells enhanced CaM KK association with 14-3-3 regardless of E2 treatment of cells (Fig. 4d). Immunoprecipitation of endogenous CaM KK also revealed an increased binding of endogenous 14-3-3 with both Forskolin and Vit D treatments (data not shown). These data suggest that Vit D is capable of promoting PKA-directed phosphorylation of CaM KK and its association with 14-3-3.

\subsection{PKA Mediates Vitamin D Inhibition of CaM Kinase Signaling}

Vit D is capable of activating rapid signaling pathways and has been proposed to stimulate cAMP/PKA, $\mathrm{PLC}$, and $\mathrm{IP}_{3}$ although the effects of these pathways exhibit cell type specific differences [11, 64]. Our data suggests that Vit D is capable of activating cAMP and PKA to block CaM KK signaling therefore we wanted to determine if Vit D treatment of cells was capable of elevating cAMP levels in our model system. Treatment of MCF-7 cells with Forskolin (Forsk) and Epi both stimulated an increase in cAMP levels within 5 minutes as expected, interestingly Vit D also significantly elevated cAMP levels in our cells (Fig. 5a). Forskolin elevates cAMP, triggers CaM KK phosphorylation, and inhibits CaM KK activity to determine if PKA was mediating these effects we utilized the selective inhibitor of PKA, H89. E2 treatment of cells did not promote CaM KK phosphorylation in contrast Forskolin stimulated a 4-fold increase in phosphorylation that was prevented by pretreating cells with H-89 (Fig. 5b). We also examined whether PKA was necessary for Vit D's ability to phosphorylate and inhibit CaM KK and as can be seen in figure 5c, Vit D stimulated CaM KK phosphorylation that was completely reversed by blocking PKA with H-89. In order to determine if cAMP and PKA block CaM KK and CaM KI signaling to ERK cells were stimulated with E2 in the presence or absence of Forskolin and Vit D followed by pretreatment with H-89. Forskolin and Vit D both inhibited CaM KK activation of ERK as can be seen previously in figures $3 \mathrm{~b}$ and $3 \mathrm{~d}$ however pretreatment of cells with H-89 prevented Forskolin from blocking E2 activation of ERK (Fig. 5d). Similarly, there were no significant differences between E2's ability to activate ERK in the presence of Vit D when cells had been incubated with the PKA 
inhibitor compound (Fig. 5e). The results suggest that both Forskolin and Vit D are capable of increasing cAMP leading to PKA's phosphorylation and inhibition of CaM KK in MCF-7 cells.

PKA has been shown to inhibit CaM KK activity through direct phosphorylation of several key CaM KK residues including serine 74 (S74), threonine 108 (T108), and serine 458 (S458) and mutation of these sites prevents phosphorylation, 14-3-3 binding, and inhibition of CaM KK activity [50]. To evaluate if Forskolin and Vit D were specifically inhibiting CaM KK through PKA's phosphorylation of S74/T108/S458 we transfected MCF-7 cells with either wild type $(w t)$ or mutant $(m t)$ forms of CaM KK. The $m t$ Flag-tagged CaM KK construct contained the following amino acid substitutions S74A, T108G, and S458A and was otherwise identical to the $w t$ Flag-tagged CaM KK. Similar to endogenous CaM KK protein, stimulation of wt-transfected cells with E2 alone did not affect CaM KK phosphorylation (Fig. 6a). Treatment of cells with Forskolin in the presence of E2 significantly increased PKA-directed phosphorylation of CaM KK (Fig. 6a). CaM KK lacking the PKA phosphorylation sites ( $m t$ CaM KK) was highly expressed in our transfected cells (Fig. 6b, lower panel) however neither Forskolin- nor E2stimulated CaM KK phosphorylation by PKA (Fig. 6b). To ensure that we were activating endogenous PKA in our system in the presence of $m t$ CaM KK whole cell lysates were analyzed by SDS-PAGE and western blotting using the phospho-PKA substrate antibody. Importantly, Forskolin treatment of MCF7 cells increased PKA activity as can be seen by increased phosphorylation of multiple bands (Fig. 6c, arrows). Vit D treatment of MCF-7 cells in the presence or absence of E2 significantly increased PKA's phosphorylation of $w t$ CaM KK while E2 alone had no effect (Fig. 6d). MCF-7 cells transfected with $m t$ CaM KK and treated with E2, Vit D, or Vit D then E2 had no effect on PKA-dependent phosphorylation of CaM KK (Fig. 6e) although Vit D stimulated an increase in cAMP (Fig. 5a) and PKA activity (data not shown). Taken together, the above data would suggest that Forskolin and Vit D are capable of utilizing PKA to specifically phosphorylate CaM KK on S74/T108/S458 residues and inhibit E2's activation of CaM KK in MCF-7 cells.

\section{Discussion and Conclusion}

In the present study we provide data to suggest that the hormone E2 rapidly stimulates the activation of a CaM KK, CaM KI, and ERK signaling pathway in MCF-7 cells. CaM KK and ERK appear to be required for the phosphorylation and induction of SRE-directed gene expression by the transcription factors, SRF and Elk-1 downstream of hormone stimulation (Fig. 7). In contrast, treatment of cells with the hormone Vit D blocked E2's ability to induce CaM KK and ERK activation as well as Elk-1/SREdependent luciferase activity. The cAMP and PKA elevating agent, Forskolin, mimicked treatment of cells with Vit D and both compounds stimulated increased phosphorylation of CaM KK and its association with the inhibitory protein, 14-3-3 (Fig. 7). The inhibitory phosphorylation of CaM KK and subsequent blockade of ERK activation requires PKA as both observations are inhibited by H-89 and occur on the previously identified PKA-target residues S74, T108 and S458. To the best of our knowledge this is the first study to demonstrate a possible mechanism for Vit D's inhibition of E2 signaling in breast cancer cells.

The CaM Kinase family of enzymes have been well established to participate in various physiological and developmental functions including hematopoiesis, neurogenesis, and metabolism [38, 41, 65-67]. The role for the different isoforms of CaM Kinases in oncogenesis is rapidly emerging and evidence supports the involvement for $\mathrm{CaM} \mathrm{KK}$, CaM KI, CaM KIV, CaM KII as well as their various substrates and cellular targets in cancer development [60,68-74]. Consistent with our data, Iglewski et al. demonstrated that urotensin treatment of primary smooth muscle cells activated a CaM KK/CaM KI/ERK proliferation pathway although the ability of other hormones to inhibit this process was not examined [75]. Similarly, recent data suggests that inhibiting CaM KK reduced both ERK and AKT activation as well as gastric cancer cell growth [76]. We did not evaluate the role of AKT or AMPK in our system although they may contribute to cell growth and survival downstream of CaM KK in several cancer cell types including breast, prostate, and myeloid [60, 70, 77, 78]. Future studies may examine the specific effect that E2 has on AKT or AMPK activation and weather Vit D impairs their phosphorylation in breast cancer cells. Interestingly, E2 control of CaM KK and CaM KI have also been implicated in the migration of medulloblastoma cells through the small $\mathrm{G}$ protein Rac1 and the scaffold protein betaPIX although the role of ERK, AKT and AMPK were not examined in that system [72]. Several small G 
proteins including Ras have been suggested to link CaM Kinase activation to downstream targets such as ERK however the precise connection among these enzymes requires further evaluation in MCF-7 cells.

CaM KII is enriched in the nervous system and plays a critical role in synaptic plasticity however it has also been implicated in the control of various cancer cells including the PC3 (prostate) type, MDAMB-231 (breast), MCF-7, A549 (lung) and MG-63 (bone) among others [79, 80]. CaM KII activation may lead to apoptosis in MCF-7 and MDA-MB-231 breast cancer cells and its phosphorylation status does not appear to change during the cell cycle in these cancer cells [81-83]. CaM KII activity has also been shown to utilize both ERK-dependent and -independent mechanisms depending on the type of cell and mode of kinase activation. CaM KII function is often identified using the inhibitor KN-93 a compound that blocks all CaM Kinase activity whereas STO-609 selectively inhibits CaM KK [56, 84]. We have previously shown that KN-93 as well as STO-609 blocks ERK activation in MCF-7 cells [31] however here we provide data using siRNA against CaM KK/KI to specifically block phosphorylation and transcriptional control of Elk-1 and SRF. Since CaM KII has been reported to play a role in MCF-7 cells future studies may focus on determining if E2 utilizes CaM KII and if so at what time point(s). In addition, the ability of E2 and Vit D to regulate the activation or inhibition of CaM Kinases, such as CaM KII, may depend on their subcellular localization, phosphorylation state and association with additional proteins.

The binding of 14-3-3 to CaM KK following its phosphorylation by PKA provides a unique inhibitory mechanism for Vit D in MCF-7 cells. 14-3-3 is a ubiquitously expressed protein with multiple isoforms that can enhance or inhibit target enzyme function as well as play a vital role in health and disease. 143-3 expression is controlled through several mechanisms and in MCF-7 cells appears to be regulated in part through the tumor suppressor p53 leading to apoptosis and growth suppression [85]. 14-3-3 inactivation or reduced protein expression appears to correlate with breast cancer progression although the precise mechanisms for 14-3-3 inhibition of E2-stimulated growth have yet to be elucidated [86]. Our results build on the observation that 14-3-3 can bind to CaM KK and inhibit its activity and extend them to include this as one way in which Vit D may inhibit CaM KK activation of ERK and transcription in breast cancer cells [50]. 14-3-3 appears to be sufficient to inhibit CaM KK following Vit $\mathrm{D}$ activation of PKA in our cells however we did not determine the requirement for 14-3-3 in this process as there are at least 7 different isoforms of the protein. It has previously been suggested that 143-3 is the primary isoform to bind CaM KK which is consistent with our observations although future studies may determine if other isoforms can bind CaM KK in MCF-7 cells [50].

The ability of CaM KK to associate with 14-3-3 or other proteins may be cell type specific and its cellular localization through post-translational modification or anchoring may also contribute to its activity. Calcium/calmodulin and CaM Kinases have been shown to interact with a variety of proteins including betaPIX, protocadherin-gC5, alphaKAP and A-Kinase Anchoring Proteins (AKAPs) [87-90]. The dynamic regulation of CaM Kinase activity by E2 and Vit D may in part be attributed to kinase association with scaffolding proteins and other regulatory proteins. For example, betaPIX has been shown to form a complex that links calcium activation of CaM KK/KI activation to the activation of Rac1 and PAK in neurons and cancer cells $[72,88]$. In contrast, if phosphatases associate with anchoring proteins in complex with CaM Kinases then this may also provide an additional mechanism of control for the pathways we have suggested here. Several phosphatases exist that function to counteract the work of CaM Kinases including Protein Phosphatase 1 (PP1), PP2, Calcineurin and CaM KP [87, 91, 92]. The specific ability of phosphatases to reverse CaM KK signaling in breast cancer cells downstream of Vit D and other hormones is an intriguing area for future studies. Alternatively, since PKA has been shown to phosphorylate and inhibit CaM KK one possible mechanism for the control of CaM KK activity and associated proteins including 14-3-3 is through AKAPs [62]. It could be hypothesized that E2 and Vit D converge on CaM KK signaling in our system through the work of AKAP-mediated signaling complexes involving PKA. AKAPs are critical modulators of cAMP and PKA signaling and have been shown to intersect with a myriad of signaling pathways including calcium/calmodulin although the specific role of AKAPs in breast cancer signaling and control of transcription requires further evaluation [90].

Our data suggests that Vit D is utilizing a novel pathway through PKA to block CaM KK activation of ERK as well as the phosphorylation of the transcription factors Elk-1 and SRF. The ability of Vit D to inhibit ERK activation in cancer cells has been previously suggested however our data extends these findings to provide a possible mechanism through PKA and CaM KK [29]. Vit D exerts its cellular 
effects upon binding its receptor(s) resulting in both rapid signaling and nuclear receptor control of transcription. Interestingly, work by Richard et al has demonstrated that Vit D specifically utilizes the $1,25 \mathrm{D}_{3}$-membrane-associated rapid response steroid-binding (MARRS) receptor in MCF-7 cells to inhibit cell proliferation although the effect of Vit D on estrogen activation of CaM KK and ERK was not evaluated [9]. Similarly, the Vit D analog MART-10 was recently shown to impair MCF-7 growth as well as the ability of VEGF to activate AKT, ERK and angiogenesis in endothelial cells [93, 94]. Vit D has been proposed to regulate several intracellular enzymes including PKC, PLC, adenylyl cyclase/cAMP/PKA, and Src in osteoblasts, chondrocytes, skeletal muscle, colon, prostate and breast cells $[10,11,95,96]$. Our results support these findings and extend them to provide mechanistic insight into the ability of Vit D to utilize cAMP/PKA to specifically phosphorylate and inhibit CaM KK function in MCF-7 cells. Future studies could also be conducted to evaluate the ability of MARRS and MART-10 to impair E2 activation of CaM KK, ERK and cell growth in our system.

The role of Vit D in health and disease is a critical area of investigation as a myriad of studies have linked Vit D levels, Vit D receptor expression, and genetic alteration of Vit D to cancer risk [97, 98] The vital role of Vit D in breast cancer risk and progresssion has been discussed for many years and reviewed recently [99] although the precise way(s) that Vit D functions varies among the types of cells, treatments and stages of the disease. The loss of Vit D receptors as well as Vit D deficiency also correlates strongly with breast cancer progression however the precise intracellular functions for the hormone and its regulation of E2 signaling require further evaluation [100, 101]. Taken together, our study suggests that Vit D activates PKA that specifically phosphorylates CaM KK on previously identified sites resulting in its association with 14-3-3 and blockade of downstream signaling in MCF-7 cells (Fig. 7). Since E2 activates CaM KK, ERK and the transcription factors Elk-1 and SRF resulting in up-regulation of gene expression our findings elucidate a possible mechanism for Vit D to inhibit breast cancer progression.

\section{Figures}
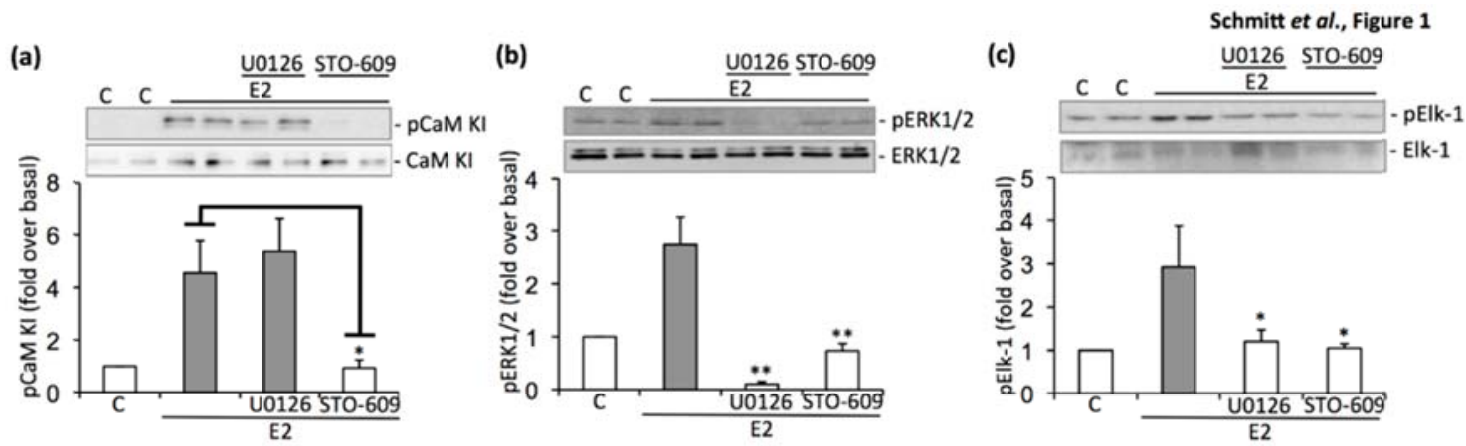

(d)
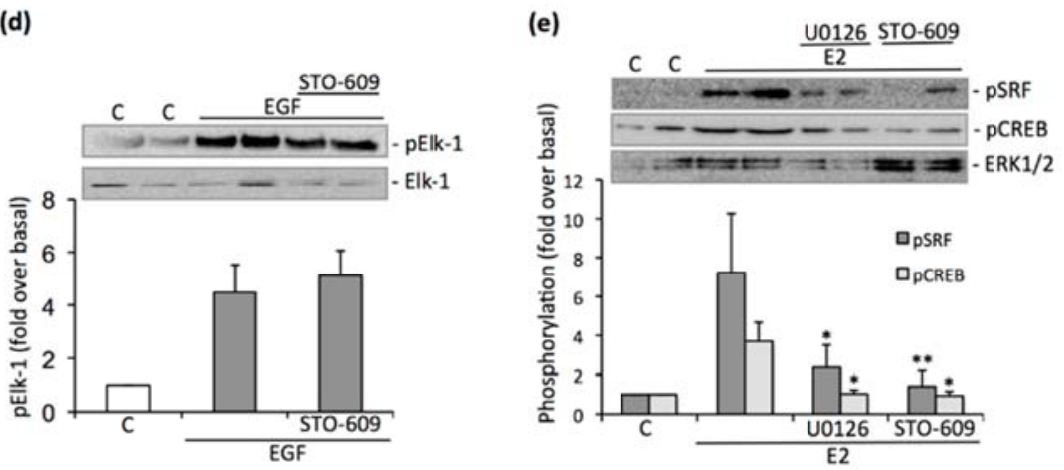

Figure 1. Estrogen activates CaM KK and ERK in MCF-7 cells a, Estrogen (E2) stimulates CaM KI phosphorylation. MCF-7 cells were treated with E2 (10nM) for 5 minutes in the presence or absence of either the U0126 $(10 \mu \mathrm{M})$ or STO-609 $(5 \mu \mathrm{M})$ compounds as described in the "materials and methods.". Endogenous CaM KI 
phosphorylation and activation was analyzed and measured by western blotting for phosphorylated-CaM KI (pCaM $\mathrm{KI}$ ). The letter "C" represents the control non-stimulated cells in all figures. The lower panel is a control western blot from cell extracts visualizing total endogenous CaM KI protein (CaM KI) used for pCaM KI analysis. Data is presented as fold CaM KI phosphorylation over control $(\mathrm{N}=6, \pm$ S.E., "*" $=\mathrm{p} \leq 0.05)$. b, E2 activates ERK through CaM KK and MEK. MCF-7 cells were stimulated with E2 for 5 minutes or pretreated with the CaM KK inhibitor STO-609 $(5 \mu \mathrm{M})$ or the MEK inhibitor U0126 $(10 \mu \mathrm{M})$ as indicated and ERK phosphorylation and activation was measured by western blotting similar to CaM KI in panel a $(\mathrm{N}=6$, \pm S.E., "**" $=\mathrm{p} \leq 0.01)$. c, Inhibition of MEK/ERK and CaM KK blocks Elk-1 phosphorylation. MCF-7 cells were treatted identically to figures $\mathrm{a}$ and $\mathrm{b}$ and analyzed for Elk-1 phosphorylation (Serine 383) by western blotting (N=6, \pm S.E.). d, STO-609 does not inhibit epidermal growth factor (EGF) phosphorylation of Elk-1 in MCF-7 cells. MCF-7 cells received no pretreatment or were pretreated with STO-609 $(5 \mu \mathrm{M})$ and then stimulated with EGF (300ng/ml) for 5 minutes $(\mathrm{N}=6, \pm$ S.E. $)$. e, E2 stimulates SRF phosphorylation via CaM KK and ERK. MCF-7 cells were treated identically to figures a-c and cell lysates were analyzed for SRF (Serine 103) and CREB (Serine 133) phosphorylation $(\mathrm{N}=6$, \pm S.E.).

(a)

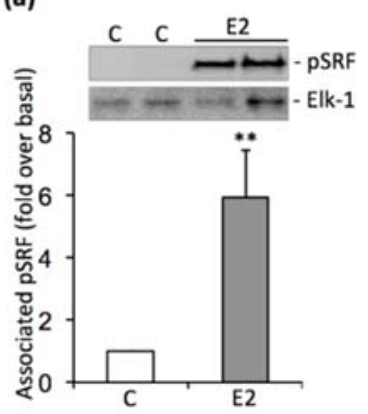

(d)

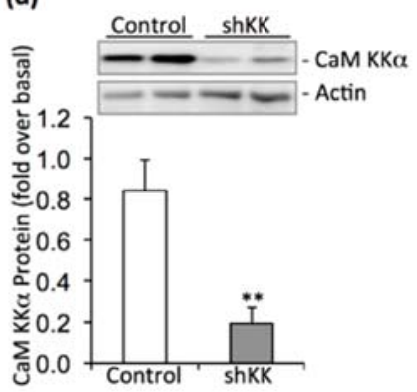

(b)

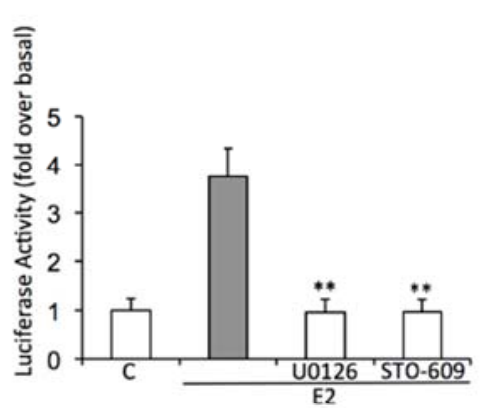

(e)

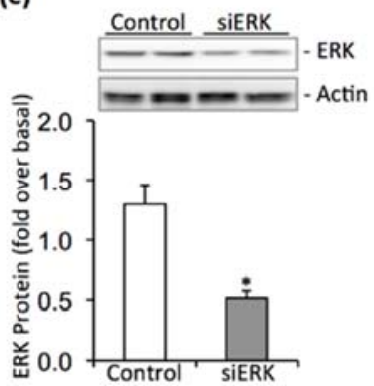

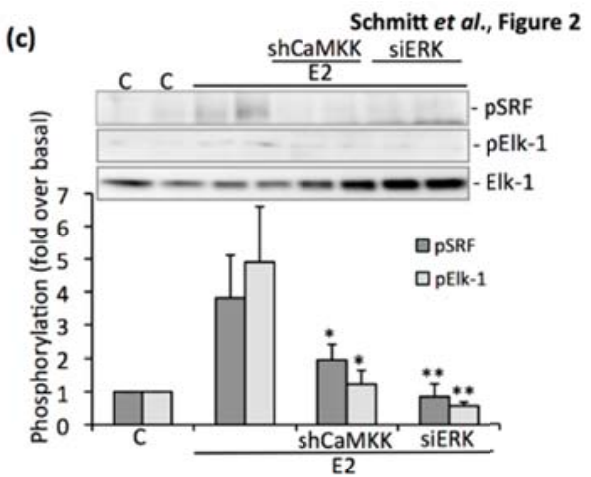

(f)

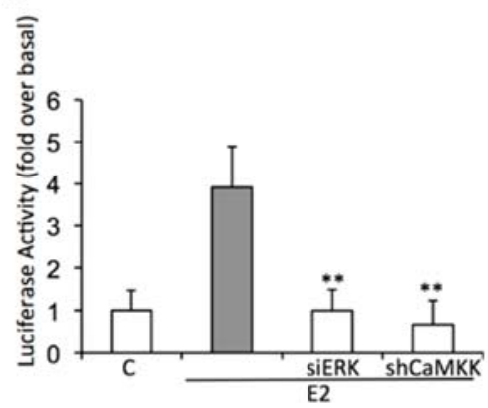

Figure 2. Elk-1 activation utilizes CaM KK and ERK a, E2 treatment of MCF-7 cells for 5 minutes induces endogenous SRF and Elk-1 association. Phospho-SRF binds Elk-1 following a 5-minute treatment of cells with 10 nM E2 followed by immunoprecipitation of Elk-1 as described in the methods (N=6, \pm S.E..). b, E2 stimulates Elk1- and SRF-dependent luciferase activity through CaM KK and ERK. SRE-transfected MCF-7 cells were pretreated with U0126 and STO-609 as in figure 1 and luciferase activity measured as described in the methods (N=8, \pm S.E.). c, Elk-1 and SRF phosphorylation requires CaM KK and ERK in MCF-7 cells. Cells transfected with shRNA directed against CaM KK or siRNA against ERK and stimulated with E2 as in panel b inhibits Elk-1 and SRF phosphorylation ( $\mathrm{N}=8$, \pm S.E.) d, shCaM KK blocks CaM KK expression. MCF-7 cells were transfected with either control plasmid or shRNA as described in the "materials and methods" and CaM KK expression was measured by western blotting and normalized to total actin, and analyzed. Data is presented as fold CaM KK expression over actin $(\mathrm{N}=8, \pm$ S.E.). e, siRNA to ERK inhibits protein expression in MCF-7 cells. MCF-7 cells were transfected with either control scrambled siRNAs or siERK as described in the "materials and methods" and ERK expression was measured by western blotting and normalized to total actin. Data is presented as folld ERK expression over actin $(\mathrm{N}=8, \pm$ S.E.). f, Elk-1-dependent transcription utilizes CaM KK and ERK downistream of 5 minute E2 stimulation. Cells were transfected as in panel c along with the SRE-reporter plasmid and luciferase activity measured and analyzed following a 5 minute $\mathrm{E} 2(10 \mathrm{nM})$ stimulation $(\mathrm{N}=8, \pm$ S.E. $)$. 
(a)

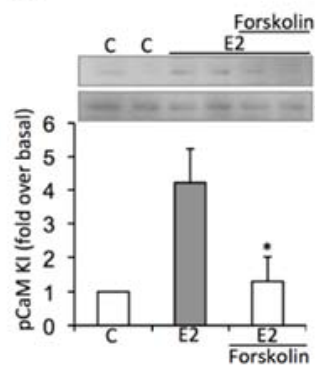

(b)

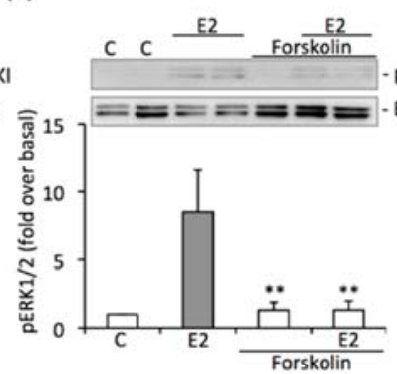

(c)

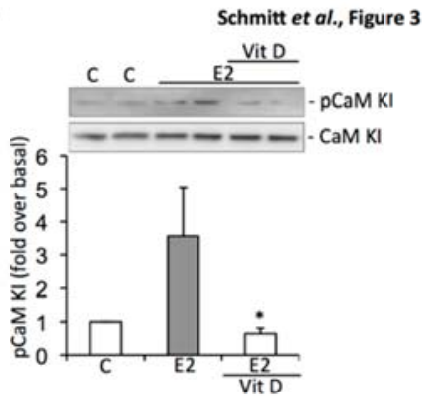

(d)

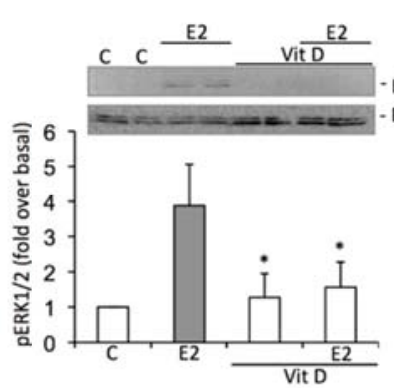

(e) Time (min) $\frac{C}{0} \frac{E 2}{5153060} \frac{\text { Vit D }}{5153060} \frac{E 2 / V i t D}{5153060}$ (f)

Figure 3. Vitamin D inhibits CaM KK, ERK and transcription in MCF-7 cells a, cAMP blocks CaM Kinase activity in MCF-7 cells. Cells received no pretreatment or were pretreated with Forskolin $(10 \mu \mathrm{M})$ and then stimulated with E2 (10nM) for 5 minutes. Endogenous CaM KI phosphorylation was measured by western blotting, normalized and quantitated as described in the methods $(\mathrm{N}=6, \pm$ S.E.). b, Forskolin inhibits CaM Kinase activation of ERK. MCF-7 cells were treated similarly to panel a and endogenous ERK phosphorylation was measured and analyzed by western blot $(\mathrm{N}=6, \pm$ S.E.). c, Vitamin D (Vit D) treatment of MCF-7 cells blocks E2 activation of CaM KK. Cells received no pretreatment or were pretreated with Vit D (10nM) and then stimulated with E2 (10nM) for 5 minutes. Endogenous CaM KI phosphorylation was measured by western blotting and quantitated as in panel a $(\mathrm{N}=6, \pm$ S.E.). d, Vit D inhibits E2 activation of ERK. Cells were treated identically to panel $\mathbf{c}$ and endogenous ERK phosphorylation was measured by western blotting and quantitated as in panel $\mathbf{c}$ $(\mathrm{N}=6, \pm \mathrm{S}$.E.). e, Sustained activation of ERK and Elk-1 by E2 is inhibited by Vit D in MCF-7 cells. Cells received no pretreatment or were pretreated with Vit D (10nM) and then E2 (10nM) or E2 (10nM) alone for the indicated times and endogenous ERK and Elk-1 phosphorylation were measured by western blotting and quantitated $(\mathrm{N}=4$, \pm S.E.). f, Vit D and Epinephrine (Epi) block E2 stimulated Elk-1-dependent luciferase activity. MCF-7 cells were transfected with the SRE-luciferase assay reporter and luciferase activity measured and analyzed following cell treatments. Serum-starved cells were left untreated or pretreated with Vit D (10nM), or Epi (10nM) and then stimulated with E2 (10nM) as indicated $(\mathrm{N}=8, \pm$ S.E.). 
(a)

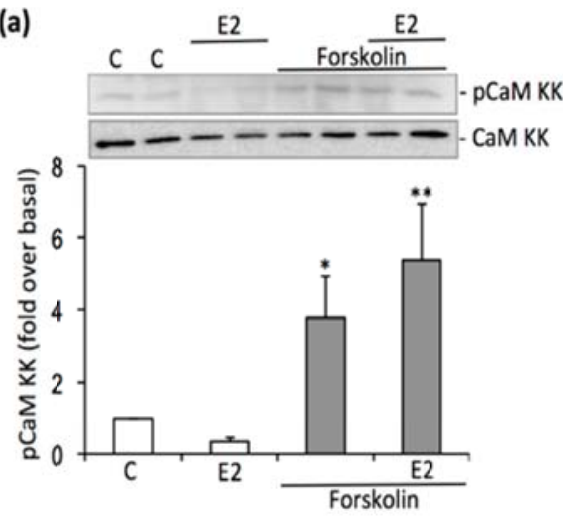

(c)

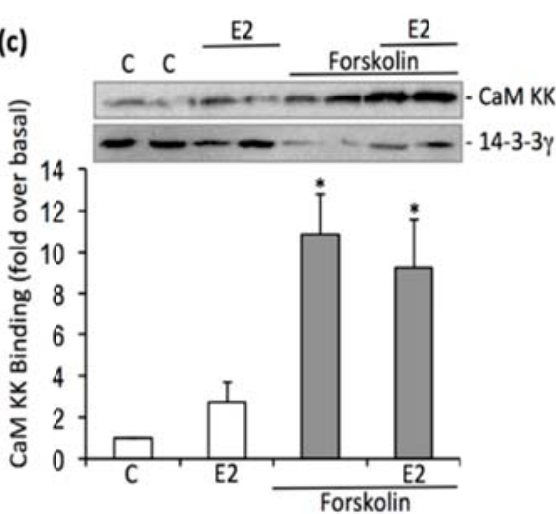

(b)

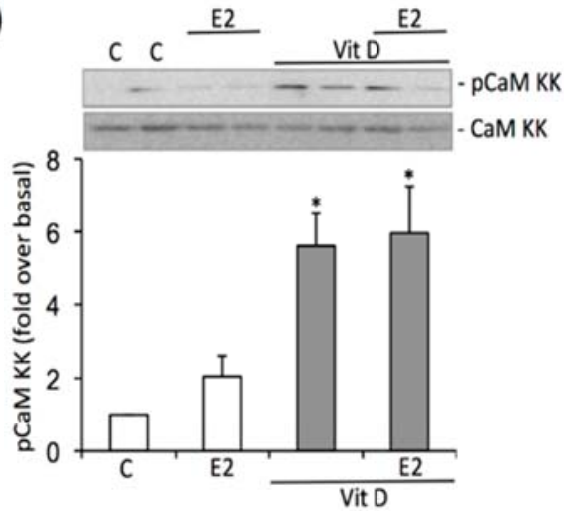

(d)

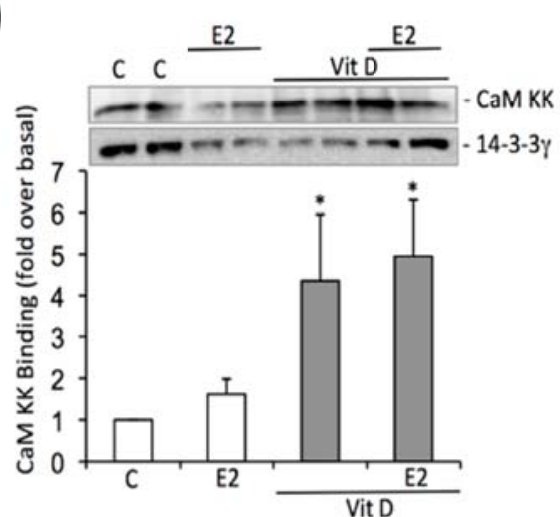

Schmitt et al., Figure 4

Figure 4. Vitamin D induces CaM KK phosphorylation and its association with 14-3-3 a, Forskolin stimulation of MCF-7 cells induces PKA-directed phosphorylation of CaM KK. MCF-7 cells received no pretreatment or were treated with E2 $(10 \mathrm{nM})$, Forskolin $(10 \mu \mathrm{M})$, or Forskolin and then E2 as indicated. Endogenous CaM KK was immunoprecipitated, western blotted, and probed with the phosphor-PKA substrate antibody as described in the methods. CaM KK phosphorylation was then quantified and analyzed (N=6, \pm S.E.). b, Vit D promotes PKA phosphorylation of CaM KK. MCF-7 cells received no pretreatment or were treated with E2 (10nM), Vit D (10nM), or Vit D and then E2 as indicated. Endogenous CaM KK was immunoprecipitated and analyzed as in panel a $(\mathrm{N}=6$, \pm S.E.). c, Forskolin enhances CaM KK binding to 14-3-3 in MCF-7 cells. MCF-7 cells were treated identically to panel a and endogenous 14-3-3 was immunoprecipitated and CaM KK association was measured by western blotting as described in the methods $(\mathrm{N}=6, \pm$ S.E.). d, Vit D stimulates CaM KK association to 14-3-3 in MCF-7 cells. MCF-7 cells were treated identically to panel $\mathbf{b}$ and endogenous 14-3-3 association with CaM KK was measured by western blotting as in panel $\mathbf{c}(\mathrm{N}=6, \pm \mathrm{S}$.E.). 
(a)

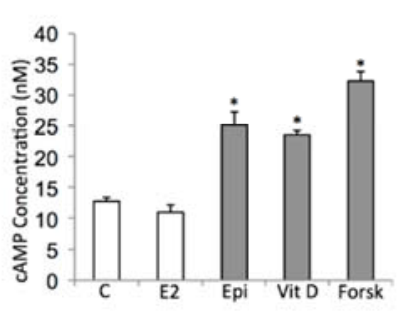

(d)

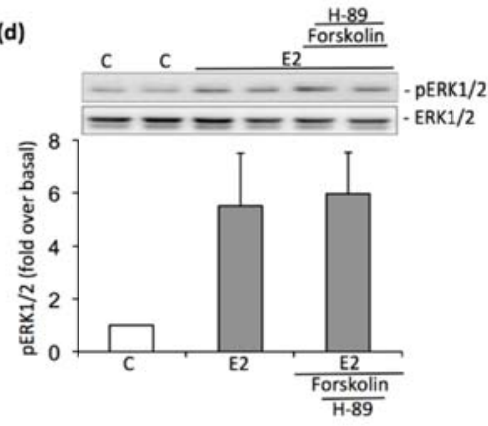

(b)

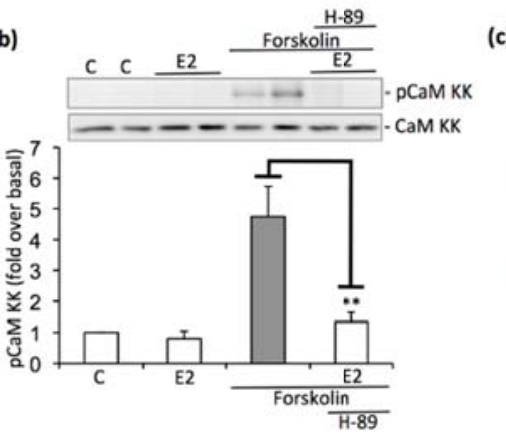

(e)

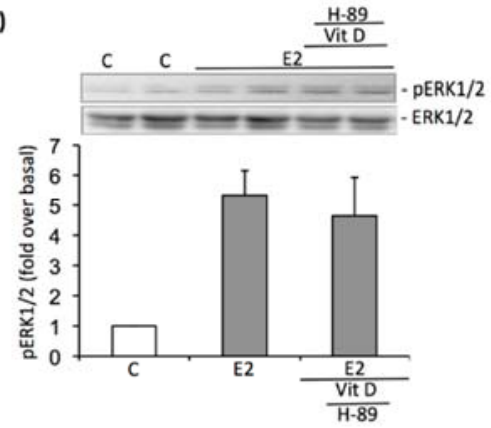

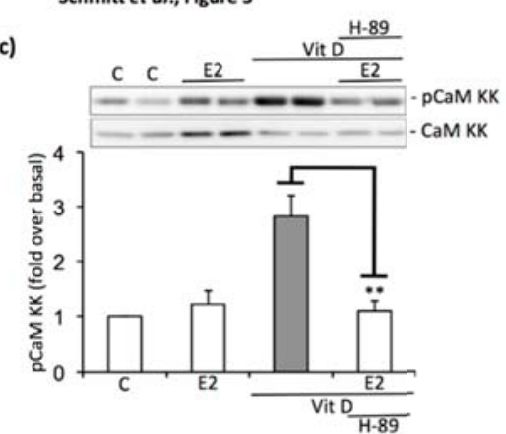

Figure 5. Vitamin D inhibits CaM KK and ERK through cAMP and PKA a, Vit D, Epi, and Forskolin (Forsk) stimulate increases in cAMP concentration in MCF-7 cells. Serum-starved cellsi were treated with E2 $(10 \mathrm{nM})$, Epi $(10 \mathrm{nM})$, Vit D $(10 \mathrm{nM})$ or Forsk $(10 \mu \mathrm{M})$ for 5 minutes as indicated. cAMP concentration was then analyzed using an ELISA assay as described in the "materials and methods" ( $\mathrm{N}=8, \pm$ S.E.). b, Forskolin treatment of MCF-7 cells stimulates phosphorylation of CaM KK through PKA. Serum-starved MCF-7 cells received no pretreatment or were pretreated with H-89 $(10 \mu \mathrm{M})$ for 30 minutes and then stimulated with E2 (10nM), Forskolin $(10 \mu \mathrm{M})$, or Forskolin and then E2 as indicated. Endogenous CaM KK was immunoprecipitated, western blotted, and probed with the phospho-PKA substrate antibody as done in figure 4. CaM KK phosphorylation was then quantified and analyzed $(\mathrm{N}=6, \pm$ S.E.). c, Vit D utilizes PKA to phosphorylate and inhibit CaM KK. MCF-7 cells were pretreated similarly to panel b and then treated with E2 (10nM), Vit D (10nM), or Vit D and then E2 as indicated and analyzed for PKA phosphorylation of CaM KK $(\mathrm{N}=6, \pm$ S.E.). d, Forskolin inhibition of ERK is prevented by the PKA inhibitor, H-89. Serum-starved MCF-7 cells were pretreated with H-89 (10pM) for 30 minutes and then stimulated with $\mathrm{E} 2(10 \mathrm{nM})$ or Forskolin $(10 \mu \mathrm{M})$ and then E2 as indicated. Endogenous ERK phosphorylation was measured by western blotting, normalized to total ERK and quantitated $(\mathrm{N}=6$, \pm S.E. $)$. e, Vit

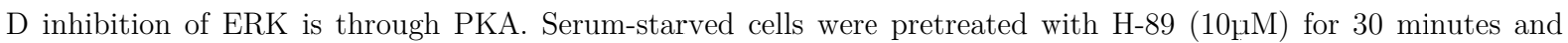
then stimulated with stimulated with E2 $(10 \mathrm{nM})$ or Vit D $(10 \mathrm{nM})$ and then E2 as indicated. Endogenous ERK phosphorylation was measured by western blotting, normalized to total ERK and analyzed $(\mathrm{N}=6$, \pm S.E. $)$. 

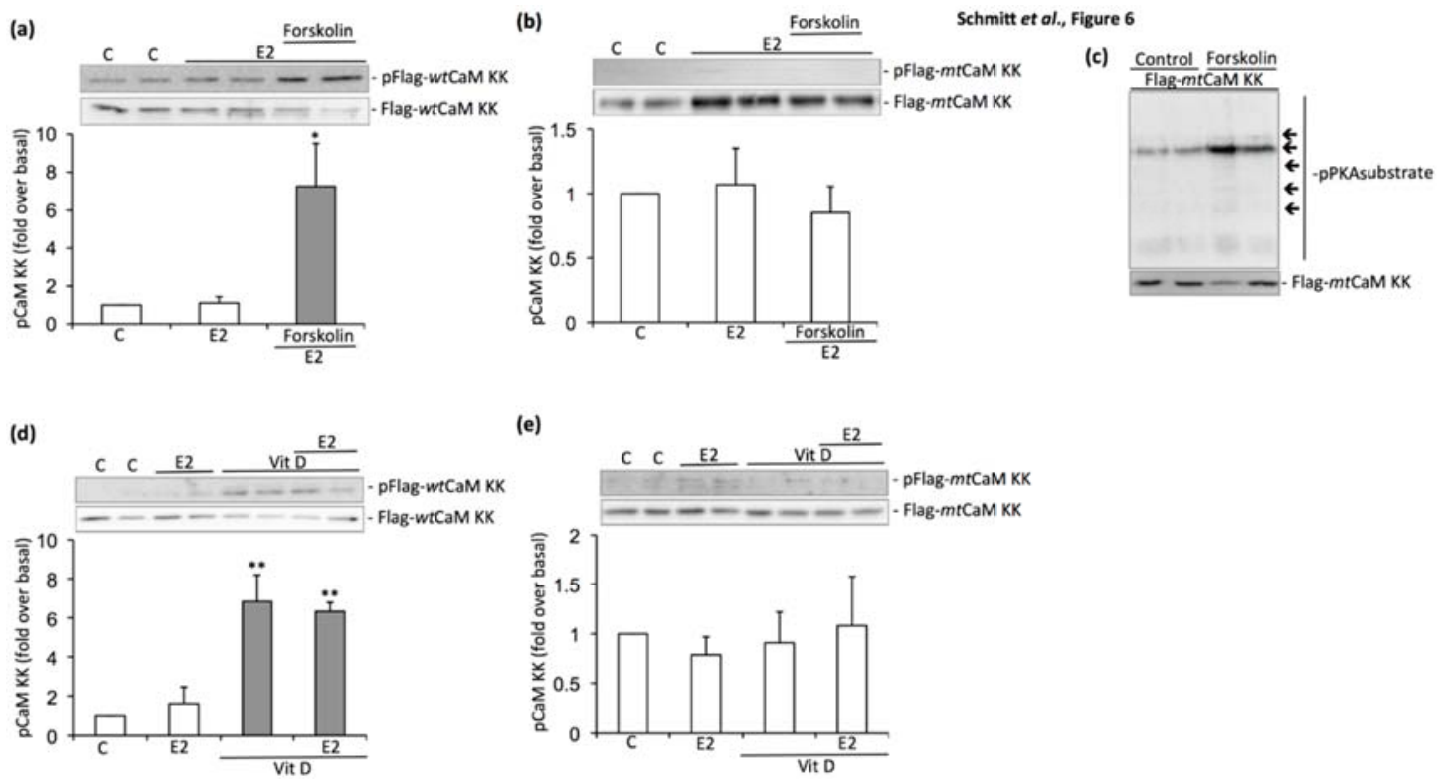

Figure 6. Forskolin and Vitamin D promote PKA site-specific inhibitory phosphorylation of CaM KK. a, Forskolin stimulates phosphorylation of wild type $(w t)$ CaM KK. MCF-7 cells were transfected with

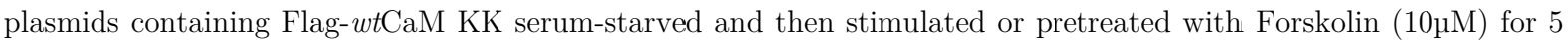
minutes and then stimulated with E2 (10nM) for 5 minutes as indicated. Lysates were then immunoprecipitated with Flag antibodies, and analyzed by western blot using the phospho-PKA substrate antibody (upper panel) or whole cell lysates were analyzed for total Flag-wtCaM KK as a loading and transfection control (lower panel) as described in the methods $(\mathrm{N}=6, \pm$ S.E.). b, PKA does not phosphorylate mutant $(m t)$ CaM KK in MCF-7 cells. Cells were transfected with plasmids containing Flag-mtCaM KK that lacks the PKA-specific phosphorylation sites (S74, T108, S458), serum-starved and then stimulated and analyzed by western blotting identically to panel a $(\mathrm{N}=6, \pm$ S.E. $)$. c, Forskolin stimulates cAMP and PKA activity in cells expressing Flag-mtCaM KK. MCF-7 cells were transfected, serum-starved and stimulated identically to panel $\mathbf{b}$. Whole cell lysates were then analyzed by SDS-PAGE and western blotting with either the phospho-PKA substrate (upper panel) or Flag (M2) antibodies (lower panel), as indicated. d, Vit D stimulates CaM KK phosphorylation via PKA. MCF-7 cells were transfected with plasmids containing Flag-wtCaM KK serum-starved and then stimulated or pretreatedl with Vit D (10nM) for 5 minutes and then stimulated with E2 (10nM) for 5 minutes as indicated. Lysates were then immunoprecipitated with Flag antibodies, and analyzed by western blot using the phospho-PKA substrate antibody (upper panel) or whole cell lysates were analyzed for total Flag-wtCaM KK as a loading and transfection control (lower panel) as described in the methods $(\mathrm{N}=6, \pm$ S.E.). e, Vit D can not stimulate PKA phosphorylation of mutant $(m t)$ CaM KK in MCF-7 cells. Cells were transfected with plasmids containing Flag-mtCaM KK that lacks the PKA-specific phosphorylation sites (S74, T108, S458), serum-starved and then stimulated and analyzied by western blotting identically to panel $\mathbf{d}(\mathrm{N}=6, \pm \mathrm{S} . \mathrm{E}$. $)$. 


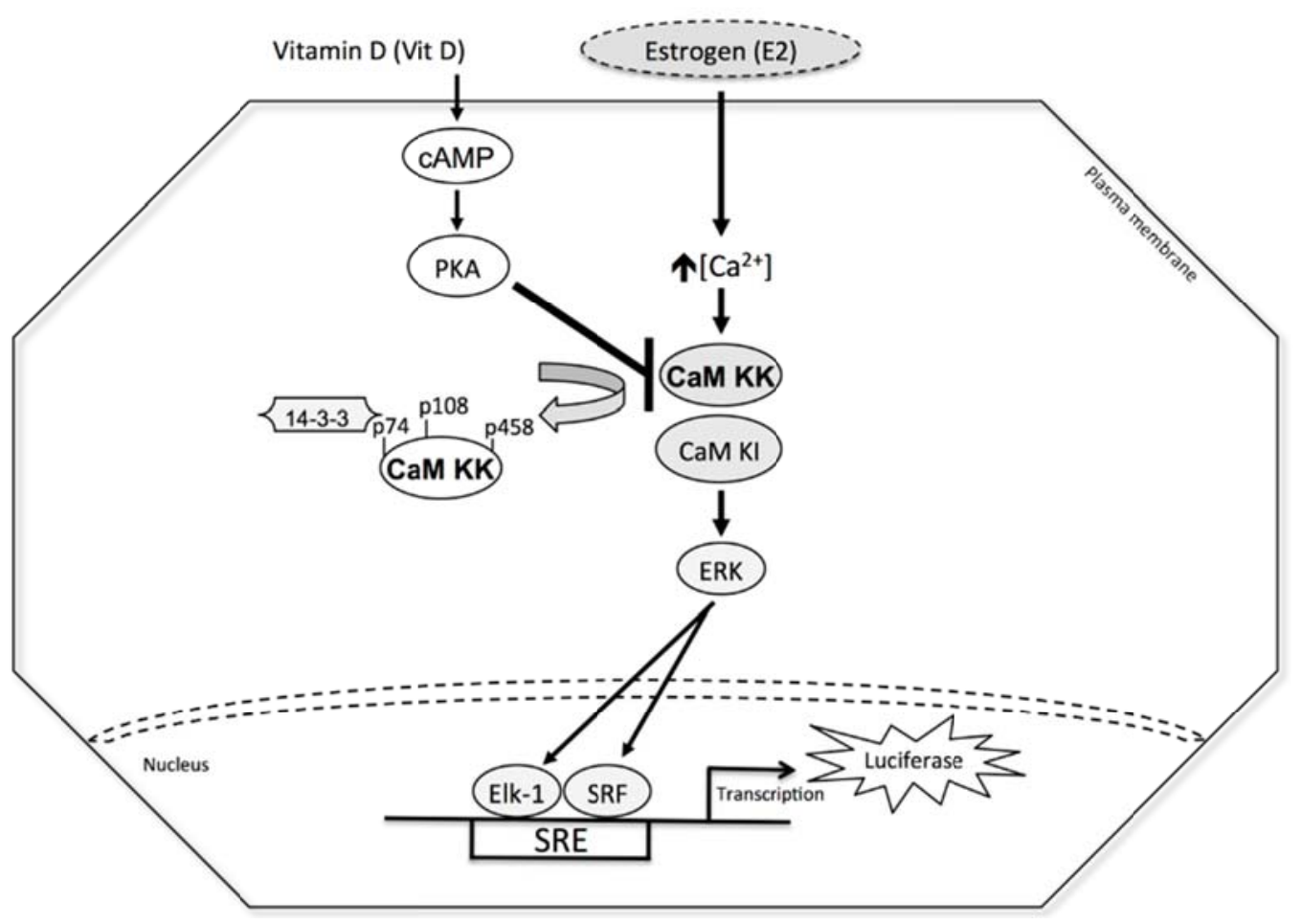

Figure 7. Proposed model for Vitamin D and PKA inhibition of CaM KK signaling in MCF-7 cells. Estrogen (E2) treatment of MCF-7 cells stimulates an increase in intracellular calcium $\left(\mathrm{Ca}^{2+}\right)$. $\mathrm{Ca}^{2+}$ binds calmodulin and activates the $\mathrm{Ca}^{2+} /$ calmodulin-dependent kinase kinase (CaM KK) and its substrate CaM KI. CaM Kinases promote ERK activation and phosphorylation of several transcription factors includling Elk-1 and SRF that bind SREs and trigger gene expression in MCF-7 cells. In contrast, Vitamin D (Vit D) and Forskolin stimulate cAMP and PKA leading to the direct phosphorylation and inhibition of CaM KK and its downstream targets including ERK and transcription factors. PKA phosphorylation and inhibition of CaM KK activity also promotes 14-3-3 association and blocks gene expression.

Acknowledgements. This work was supported in part through a grant from the M.J. Murdock Charitable Trust to J.M.S. \#2009261 and \#2011267. This work was also supporterd in part through the Jack S. Holman Endowment fund to J.M.S and the Paul K. and Evalyn E.C. Richtter Memorial Fund at George Fox University. The authors wish to thank Drs. Monika Davare and Tom Soderling at Oregon Health Sciences University (OHSU) for helpful discussions and valuable reagents.

Conflict of interest: The authors declare that they have no conflict of interest.

\section{References}

1. Fuhrman, B.J., et al., Sunlight, polymorphisms of vitamin D-related genes and risk of breast cancer. Anticancer research, 2013. 33(2): p. 543-51.

2. Garland, F.C., et al., Geographic variation in breast cancer mortality in the United States: a hypothesis involving exposure to solar radiation. Prev Med, 1990. 19(6): p. 614-22.

3. Shao, T., P. Klein, and M.L. Grossbard, Vitamin D and breast cancer. The oncologist, 2012. 17(1): p. 36-45.

4. Colston, K.W., et al., Vitamin D status and breast cancer risk. Anticancer research, 2006. 26(4A): p. 2573-80.

5. Tangpricha, V., et al., Vitamin D deficiency enhances the growth of MC-26 colon cancer xenografts in Balb/c mice. The Journal of nutrition, 2005. 135(10): p. 2350-4. 
6. Ooi, L.L., et al., Vitamin D deficiency promotes growth of MCF-7 human breast cancer in a rodent model of osteosclerotic bone metastasis. Bone, 2010. 47(4): p. 795-803.

7. Ooi, L.L., et al., Vitamin D deficiency promotes human breast cancer growth in a murine model of bone metastasis. Cancer research, 2010. 70(5): p. 1835-44.

8. Alimirah, F., et al., Functional significance of vitamin D receptor FokI polymorphism in human breast cancer cells. PLoS One, 2011. 6(1): p. e16024.

9. Richard, C.L., et al., Involvement of 1,25D3-MARRS (membrane associated, rapid response steroid-binding), a novel vitamin D receptor, in growth inhibition of breast cancer cells. Exp Cell Res, 2010. 316(5): p. 695-703.

10.Boyan, B.D., et al., Plasma membrane requirements for 1alpha,25(OH)2D3 dependent PKC signaling in chondrocytes and osteoblasts. Steroids, 2006. 71(4): p. 286-90.

11.Boland, R.L., VDR activation of intracellular signaling pathways in skeletal muscle. Mol Cell Endocrinol, 2011. 347(1-2): p. 11-6.

12.Larriba, M.J., et al., Interaction of vitamin D with membrane-based signaling pathways. Front Physiol, 2014. 5: p. 60 .

13.Slominski, A.T., et al., Novel vitamin D hydroxyderivatives inhibit melanoma growth and show differential effects on normal melanocytes. Anticancer Res, 2012. 32(9): p. 3733-42.

14.Slominski, A.T., et al., 20-Hydroxyvitamin D2 is a noncalcemic analog of vitamin D with potent antiproliferative and prodifferentiation activities in normal and malignant cells. Am J Physiol Cell Physiol, 2011. 300(3): p. C52641.

15.Kittaka, A., et al., Potent 19-norvitamin D analogs for prostate and liver cancer therapy. Future Med Chem, 2012. 4(16): p. 2049-65.

16.Chiang, K.C., et al., MART-10, a New Generation of Vitamin D Analog, Is More Potent than 1alpha,25Dihydroxyvitamin D(3) in Inhibiting Cell Proliferation and Inducing Apoptosis in ER+ MCF-7 Breast Cancer Cells. Evid Based Complement Alternat Med, 2012. 2012: p. 310872.

17.Karlsson, S., et al., Vitamin D and prostate cancer: the role of membrane initiated signaling pathways in prostate cancer progression. J Steroid Biochem Mol Biol, 2010. 121(1-2): p. 413-6.

18. Masuyama, R., et al., Vitamin D receptor in chondrocytes promotes osteoclastogenesis and regulates FGF23 production in osteoblasts. J Clin Invest, 2006. 116(12): p. 3150-9.

19.Wang, Y., J. Zhu, and H.F. DeLuca, Identification of the vitamin D receptor in osteoblasts and chondrocytes but not osteoclasts in mouse bone. J Bone Miner Res, 2014. 29(3): p. 685-92.

20.Cordes, T., et al., Modulation of MAPK ERK1 and ERK2 in VDR-positive and -negative breast cancer cell lines. Anticancer Res, 2006. 26(4A): p. 2749-53.

21.Cordes, T., et al., Expression of splice variants of 1alpha-hydroxylase in mcf-7 breast cancer cells. J Steroid Biochem Mol Biol, 2007. 103(3-5): p. 326-9.

22.Fischer, D., et al., 25-Hydroxyvitamin D3 1alpha-hydroxylase splice variants in breast cell lines MCF-7 and MCF-10. Cancer Genomics Proteomics, 2007. 4(4): p. 295-300.

23.Berg, J.P. and E. Haug, Vitamin D: a hormonal regulator of the cAMP signaling pathway. Crit Rev Biochem Mol Biol, 1999. 34(5): p. 315-23.

24.Boyan, B.D. and Z. Schwartz, Rapid vitamin D-dependent PKC signaling shares features with estrogendependent PKC signaling in cartilage and bone. Steroids, 2004. 69(8-9): p. 591-7.

25.Carlberg, C. and M.J. Campbell, Vitamin D receptor signaling mechanisms: integrated actions of a well-defined transcription factor. Steroids, 2013. 78(2): p. 127-36.

26.Gniadecki, R., Nongenomic signaling by vitamin D: a new face of Src. Biochem Pharmacol, 1998. 56(10): p. 12737.

27.Sergeev, I.N., Vitamin D and cellular Ca2+ signaling in breast cancer. Anticancer Res, 2012. 32(1): p. 299-302.

28. Brosseau, C.M., G. Pirianov, and K.W. Colston, Involvement of stress activated protein kinases (JNK and p38) in 1,25 dihydroxyvitamin D3-induced breast cell death. Steroids, 2010. 75(13-14): p. 1082-8.

29. Capiati, D.A., et al., Inhibition of serum-stimulated mitogen activated protein kinase by 1alpha,25(OH)2-vitamin D3 in MCF-7 breast cancer cells. J Cell Biochem, 2004. 93(2): p. 384-97.

30.Visram, H. and P.A. Greer, 17beta-estradiol and tamoxifen stimulate rapid and transient ERK activationin MCF-7 cells via distinct signaling mechanisms. Cancer Biol Ther, 2006. 5(12): p. 1677-82. 
31.Schmitt, J.M., et al., ERK activation and cell growth require CaM kinases in MCF-7 breast cancer cells. Mol Cell Biochem, 2010. 335(1-2): p. 155-71.

32.Liu, J.F., et al., FGF-2 and TPA induce matrix metalloproteinase-9 secretion in MCF-7 cells through PKC activation of the Ras/ERK pathway. Biochem Biophys Res Commun, 2002. 293(4): p. 1174-82.

33. Muscella, A., et al., PKC-zeta is required for angiotensin II-induced activation of ERK and synthesis of C-FOS in MCF-7 cells. J Cell Physiol, 2003. 197(1): p. 61-8.

34.Rieber, M. and M. Strasberg-Rieber, p53 inactivation decreases dependence on estrogen/ERK signalling for proliferation but promotes EMT and susceptility to 3-bromopyruvate in ERalpha+ breast cancer MCF-7 cells. Biochem Pharmacol, 2014. 88(2): p. 169-77.

35.Aksamitiene, E., et al., Prolactin-stimulated activation of ERK1/2 mitogen-activated protein kinases is controlled by PI3-kinase/Rac/PAK signaling pathway in breast cancer cells. Cell Signal, 2011. 23(11): p. 1794805 .

36.Improta-Brears, T., et al., Estrogen-induced activation of mitogen-activated protein kinase requires mobilization of intracellular calcium. Proc Natl Acad Sci U S A, 1999. 96(8): p. 4686-91.

37.Thomas, W., et al., Estrogen induces phospholipase A2 activation through ERK1/2 to mobilize intracellular calcium in MCF-7 cells. Steroids, 2006. 71(3): p. 256-65.

38.Wayman, G.A., et al., Analysis of CaM-kinase signaling in cells. Cell Calcium, 2011.

39.Colomer, J. and A.R. Means, Physiological roles of the Ca2+/CaM-dependent protein kinase cascade in health and disease. Subcell Biochem, 2007. 45: p. 169-214.

40.Skelding, K.A., J.A. Rostas, and N.M. Verrills, Controlling the cell cycle: the role of calcium/calmodulinstimulated protein kinases I and II. Cell Cycle, 2011. 10(4): p. 631-9.

41.Racioppi, L. and A.R. Means, Calcium/calmodulin-dependent protein kinase kinase 2: roles in signaling and pathophysiology. J Biol Chem, 2012. 287(38): p. 31658-65.

42.Wayman, G.A., et al., An activity-regulated microRNA controls dendritic plasticity by down-regulating p250GAP. Proc Natl Acad Sci U S A, 2008. 105(26): p. 9093-8.

43.Chang, F., et al., Signal transduction mediated by the Ras/Raf/MEK/ERK pathway from cytokine receptors to transcription factors: potential targeting for therapeutic intervention. Leukemia, 2003. 17(7): p. 1263-93.

44.Wiegert, J.S. and H. Bading, Activity-dependent calcium signaling and ERK-MAP kinases in neurons: a link to structural plasticity of the nucleus and gene transcription regulation. Cell Calcium, 2011. 49(5): p. 296-305.

45.Zhang, H.M., et al., Mitogen-induced recruitment of ERK and MSK to SRE promoter complexes by ternary complex factor Elk-1. Nucleic Acids Res, 2008. 36(8): p. 2594-607.

46.Gille, H., et al., ERK phosphorylation potentiates Elk-1-mediated ternary complex formation and transactivation. Embo J, 1995. 14(5): p. 951-62.

47.Cruzalegui, F.H., E. Cano, and R. Treisman, ERK activation induces phosphorylation of Elk-1 at multiple S/TP motifs to high stoichiometry. Oncogene, 1999. 18(56): p. 7948-57.

48.Stork, P.J. and J.M. Schmitt, Crosstalk between cAMP and MAP kinase signaling in the regulation of cell proliferation. Trends Cell Biol, 2002. 12(6): p. 258-66.

49.Ligumsky, H., et al., The peptide-hormone glucagon-like peptide-1 activates cAMP and inhibits growth of breast cancer cells. Breast Cancer Res Treat, 2012. 132(2): p. 449-61.

50.Davare, M.A., et al., Inhibition of calcium/calmodulin-dependent protein kinase kinase by protein 14-3-3. J Biol Chem, 2004. 279(50): p. 52191-9.

51.Wayman, G.A., et al., Activity-dependent dendritic arborization mediated by CaM-kinase I activation and enhanced CREB-dependent transcription of Wnt-2. Neuron, 2006. 50(6): p. 897-909.

52.Sato, K., et al., Regulation of osteoclast differentiation and function by the CaMK-CREB pathway. Nat Med, 2006. 12(12): p. 1410-6.

53.Schmitt, J.M., et al., Calcium activation of ERK mediated by calmodulin kinase I. J Biol Chem, 2004. 279(23): p. 24064-72.

54.Enslen, H., et al., Regulation of mitogen-activated protein kinases by a calcium/calmodulin-dependent protein kinase cascade. Proc Natl Acad Sci U S A, 1996. 93(20): p. 10803-8.

55.Rodriguez-Mora, O.G., et al., Calcium/calmodulin-dependent kinase I and calcium/calmodulin-dependent kinase kinase participate in the control of cell cycle progression in MCF-7 human breast cancer cells. Cancer Res, 2005. 65(12): p. 5408-16. 
56. Tokumitsu, H., et al., STO-609, a specific inhibitor of the $\mathrm{Ca}(2+) /$ calmodulin-dependent protein kinase kinase. J Biol Chem, 2002. 277(18): p. 15813-8.

57.Tokumitsu, H., et al., A single amino acid difference between alpha and beta Ca2+/calmodulin-dependent protein kinase kinase dictates sensitivity to the specific inhibitor, STO-609. J Biol Chem, 2003. 278(13): p. 10908-13.

58.Bebien, M., et al., Immediate-early gene induction by the stresses anisomycin and arsenite in human osteosarcoma cells involves MAPK cascade signaling to Elk-1, CREB and SRF. Oncogene, 2003. 22(12): p. 183647.

59.Aplin, A.E., et al., Integrin-mediated adhesion regulates ERK nuclear translocation and phosphorylation of Elk-1. J Cell Biol, 2001. 153(2): p. 273-82.

60.Schmitt, J.M., et al., CaM kinase control of AKT and LNCaP cell survival. J Cell Biochem, 2012. 113(5): p. 1514-26.

61.Evans, E.L., et al., Dimer formation and conformational flexibility ensure cytoplasmic stability and nuclear accumulation of Elk-1. Nucleic Acids Res, 2011. 39(15): p. 6390-402.

62.Wayman, G.A., H. Tokumitsu, and T.R. Soderling, Inhibitory cross-talk by cAMP kinase on the calmodulindependent protein kinase cascade. J Biol Chem, 1997. 272(26): p. 16073-6.

63.Matsushita, M. and A.C. Nairn, Inhibition of the Ca2+/calmodulin-dependent protein kinase I cascade by cAMP-dependent protein kinase. J Biol Chem, 1999. 274(15): p. 10086-93.

64.Bellido, T., et al., Evidence for the participation of protein kinase $\mathrm{C}$ and $3^{\prime}, 5^{\prime}$-cyclic AMP-dependent protein kinase in the stimulation of muscle cell proliferation by 1,25-dihydroxy-vitamin D3. Mol Cell Endocrinol, 1993. 90(2): p. 231-8.

65.Anderson, K.A., et al., Hypothalamic CaMKK2 contributes to the regulation of energy balance. Cell Metab, 2008. $7(5):$ p. $377-88$.

66.Hell, J.W., CaMKII: claiming center stage in postsynaptic function and organization. Neuron, 2014. 81(2): p. 249-65.

67.Shonesy, B.C., et al., CaMKII: a molecular substrate for synaptic plasticity and memory. Prog Mol Biol Transl Sci, 2014. 122: p. 61-87.

68.Monaco, S., et al., A novel crosstalk between calcium/calmodulin kinases II and IV regulates cell proliferation in myeloid leukemia cells. Cell Signal, 2015. 27(2): p. 204-14.

69.Lin, F., et al., The camKK2/camKIV relay is an essential regulator of hepatic cancer. Hepatology, 2015.

70.Su, R., et al., MiR-181 family: regulators of myeloid differentiation and acute myeloid leukemia as well as potential therapeutic targets. Oncogene, 2014. 0.

71.Racioppi, L., CaMKK2: a novel target for shaping the androgen-regulated tumor ecosystem. Trends Mol Med, 2013. 19(2): p. 83-8.

72.Davare, M.A., T. Saneyoshi, and T.R. Soderling, Calmodulin-kinases regulate basal and estrogen stimulated medulloblastoma migration via Rac1. J Neurooncol, 2011. 104(1): p. 65-82.

73.Zhang, Y.H., et al., Polymorphism rs7214723 in CAMKK1 and lung cancer risk in Chinese population. Tumour Biol, 2013. 34(5): p. 3147-52.

74.Daft, P.G., et al., Alpha-CaMKII plays a critical role in determining the aggressive behavior of human osteosarcoma. Mol Cancer Res, 2013. 11(4): p. 349-59.

75.Iglewski, M. and S.R. Grant, Urotensin II-induced signaling involved in proliferation of vascular smooth muscle cells. Vasc Health Risk Manag, 2010. 6: p. 723-34.

76.Ma, Z., et al., Growth inhibition of human gastric adenocarcinoma cells in vitro by STO-609 is independent of calcium/calmodulin-dependent protein kinase kinase-beta and adenosine monophosphate-activated protein kinase. Am J Transl Res, 2016. 8(2): p. 1164-71.

77.Deshmukh, R.R. and Q.P. Dou, Proteasome inhibitors induce AMPK activation via CaMKKbeta in human breast cancer cells. Breast Cancer Res Treat, 2015. 153(1): p. 79-88.

78.Popovics, P., et al., Targeting the 5'-AMP-activated protein kinase and related metabolic pathways for the treatment of prostate cancer. Expert Opin Ther Targets, 2015. 19(5): p. 617-32.

79. Mamaeva, O.A., et al., Calcium/calmodulin-dependent kinase II regulates notch-1 signaling in prostate cancer cells. J Cell Biochem, 2009. 106(1): p. 25-32. 
80.Chai, S., et al., Ca2+/calmodulin-dependent protein kinase IIgamma enhances stem-like traits and tumorigenicity of lung cancer cells. Oncotarget, 2015. 6(18): p. 16069-83.

81.Hoffman, A., et al., Dephosphorylation of CaMKII at T253 controls the metaphase-anaphase transition. Cell Signal, 2014. 26(4): p. 748-56.

82. Chen, S., et al., Fucoidan induces cancer cell apoptosis by modulating the endoplasmic reticulum stress cascades. PLoS One, 2014. 9(9): p. e108157.

83.Rodriguez-Mora, O.G., et al., Inhibition of the CaM-kinases augments cell death in response to oxygen radicals and oxygen radical inducing cancer therapies in MCF-7 human breast cancer cells. Cancer Biol Ther, 2006. 5(8): p. $1022-30$.

84.Hidaka, H., M. Hagiwara, and H. Tokumitsu, Novel and selective inhibitors of CaM-kinase II and other calmodulin-dependent enzymes. Adv Exp Med Biol, 1990. 269: p. 159-62.

85.Zheng, G., et al., 14-3-3sigma regulation by p53 mediates a chemotherapy response to 5-fluorouracil in MCF-7 breast cancer cells via Akt inactivation. FEBS Lett, 2012. 586(2): p. 163-8.

86.Urano, T., et al., Efp targets 14-3-3 sigma for proteolysis and promotes breast tumour growth. Nature, 2002. 417(6891): p. 871-5.

87. Onouchi, T., et al., Regulation of $\mathrm{Ca}(2+) /$ calmodulin-dependent protein kinase phosphatase (CaMKP/PPM1F) by protocadherin-gammaC5 (Pcdh-gammaC5). Arch Biochem Biophys, 2015. 585: p. 109-20.

88.Saneyoshi, T., et al., Activity-dependent synaptogenesis: regulation by a CaM-kinase kinase/CaM-kinase I/betaPIX signaling complex. Neuron, 2008. 57(1): p. 94-107.

89.O'Leary, H., et al., Nuclear targeting of the CaMKII anchoring protein alphaKAP is regulated by alternative splicing and protein kinases. Brain Res, 2006. 1086(1): p. 17-26.

90.Woolfrey, K.M. and M.L. Dell'Acqua, Coordination of Protein Phosphorylation and Dephosphorylation in Synaptic Plasticity. J Biol Chem, 2015. 290(48): p. 28604-12.

91.Hashimoto, Y., M.M. King, and T.R. Soderling, Regulatory interactions of calmodulin-binding proteins: phosphorylation of calcineurin by autophosphorylated Ca2+/calmodulin-dependent protein kinase II. Proc Natl Acad Sci U S A, 1988. 85(18): p. 7001-5.

92.Lee, C.W., et al., Interaction between salt-inducible kinase 2 and protein phosphatase $2 \mathrm{~A}$ regulates the activity of calcium/calmodulin-dependent protein kinase I and protein phosphatase methylesterase-1. J Biol Chem, 2014. 289(30): p. 21108-19.

93.Chiang, K.C., et al., MART-10, a less calcemic vitamin D analog, is more potent than 1alpha,25dihydroxyvitamin D3 in inhibiting the metastatic potential of MCF-7 breast cancer cells in vitro. J Steroid Biochem Mol Biol, 2014. 139: p. 54-60.

94.Chiang, K.C., et al., MART-10, the new brand of 1alpha,25(OH)2D3 analog, is a potent anti-angiogenic agent in vivo and in vitro. J Steroid Biochem Mol Biol, 2016. 155(Pt A): p. 26-34.

95.Buitrago, C., M. Costabel, and R. Boland, PKC and PTPalpha participate in Src activation by 1alpha,25OH2 vitamin D3 in C2C12 skeletal muscle cells. Mol Cell Endocrinol, 2011. 339(1-2): p. 81-9.

96. Bernichtein, S., et al., Vitamin D3 Prevents Calcium-Induced Progression of Early-Stage Prostate Tumors by Counteracting TRPC6 and Calcium Sensing Receptor Upregulation. Cancer Res, 2017. 77(2): p. 355-365.

97.Berridge, M.J., Vitamin D cell signalling in health and disease. Biochem Biophys Res Commun, 2015. 460(1): p. $53-71$.

98.Christakos, S., et al., Vitamin D: beyond bone. Ann N Y Acad Sci, 2013. 1287: p. 45-58.

99.Bandera Merchan, B., et al., The role of vitamin D and VDR in carcinogenesis: Through epidemiology and basic sciences. J Steroid Biochem Mol Biol, 2017. 167: p. 203-218.

100.LaPorta, E. and J. Welsh, Modeling vitamin D actions in triple negative/basal-like breast cancer. J Steroid Biochem Mol Biol, 2014. 144 Pt A: p. 65-73.

101.Welsh, J., Vitamin D and breast cancer: insights from animal models. Am J Clin Nutr, 2004. 80(6 Suppl): p. $1721 \mathrm{~S}-4 \mathrm{~S}$ 